\title{
Geostatistics for Environmental Applications
}

\section{J. Jaime Gómez-Hernández ${ }^{1}$}

Published online: 23 November 2015

(C) International Association for Mathematical Geosciences 2015

This is a special issue comprising papers on geostatistics applied to environmental sciences and engineering. The papers deal with geothermal modeling, hydrogeology, fishery, and soil sciences, and they span from the application of multivariate indicator cokriging, to stochastic simulation and risk analysis.

The first paper by Bruno and Tinti, while focused on a geothermal application, discusses an interesting theoretical issue on how the coordinate system used to reference a regionalized variable may impact the type of geostatistical model to use.

The second paper by Junez and colleagues describes a procedure to evaluate the performance of a groundwater quality control network for the purposes of computing the vulnerability of an aquifer using the DRASTIC methodology. The method uses a formulation of the Kalman filter in a spatial context, in which measurement locations are sequentially added to the monitoring network and the estimation covariance is updated as new locations are added. Through an optimization procedure, the authors determine the set of locations that produce the minimum estimation variance.

The third paper by Bakshevskaia and Pozdniakov deals with the prediction of radioactive contamination induced by the deep injection of radioactive waste in Siberia. For this purpose, a high-resolution heterogeneous groundwater model around the disposal site is first built using geostatistical tools and then upscaled. The model results are compared with experimental observations.

The fourth paper by Petitgas and colleagues uses indicator kriging to identify the location of hotspots for fishing. The main idea is to identify yearly a top cutoff of fish abundance that can be used to separate the hotspots from the rest, and then cokrige the indicator variables resulting for each year to create hotspot maps.

J. Jaime Gómez-Hernández jgomez@upv.es

1 Institute for Water and Environmental Engineering, Universitat Politècnica de València, Camino de Vera s/n, 46022 Valencia, Spain 
The fifth paper by Gengler and Bogaert presents a new approach to mapping categorical variables, more precisely soil drainage classes using Bayesian data fusion, a simplification of the Bayesian maximum entropy approach.

And finally, the sixth paper by D'Or and Destain describes the calculation of risk maps of soil compaction through the analysis of maps of precompression stress computed with pedotransfer functions from a set of eight variables. Through the use of simulated maps, the risk that the stress induced by the use of different types of machinery is above the precompression stress of the soil is computed and mapped.

Geostatistics has found its way into environmental science and engineering much beyond the mining realm of its origins; this special issue presents a good showcase of applications in a variety of fields proving the continuous advance in environmental geostatistics. 